\title{
Vessel wall enhancement of intracranial aneurysms: fact or artifact?
}

\author{
Bart M. W. Cornelissen, MSc, ${ }^{1-3}$ Eva L. Leemans, MSc, ${ }^{1,2}$ Cornelis H. Slump, PhD, ${ }^{3}$ \\ Henk A. Marquering, PhD, ${ }^{1,2}$ Charles B. L. M. Majoie, MD, PhD, ${ }^{1}$ and René van den Berg, MD, PhD' \\ 1Department of Radiology and Nuclear Medicine, Amsterdam Neuroscience, Amsterdam UMC, University of Amsterdam; \\ 2Department of Biomedical Engineering and Physics, Amsterdam UMC, University of Amsterdam, Amsterdam; and ${ }^{3}$ University of \\ Twente, MIRA Institute for Biomedical Technology and Technical Medicine, Enschede, The Netherlands
}

\begin{abstract}
OBJECTIVE For patients with subarachnoid hemorrhage (SAH) and multiple intracranial aneurysms, it is often challenging to identify the ruptured aneurysm. Some investigators have asserted that vessel wall imaging (VWI) can be used to identify the ruptured aneurysm since wall enhancement after contrast agent injection is presumably related to inflammation in unstable and ruptured aneurysms. The aim of this study was to determine whether additional factors contribute to aneurysm wall enhancement by assessing imaging data in a series of patients.
\end{abstract}

METHODS Patients with symptoms of SAH who subsequently underwent VWI in the period between January 2017 and September 2018 were eligible for study inclusion. Three-dimensional turbo spin-echo sequences with motion-sensitized driven-equilibrium preparation pulses were acquired using a 3-T MRI scanner to visualize the aneurysm wall. Identification of the ruptured aneurysm was based on aneurysm characteristics and hemorrhage distributions on MRI. Complementary imaging data (CT, DSA, MRI) were used to assess potential underlying enhancement mechanisms. Additionally, aneurysm luminal diameter measurements on MRA were compared with those on contrast-enhanced VWI to assess the intraluminal contribution to aneurysm enhancement.

RESULTS Six patients with 14 aneurysms were included in this series. The mean aneurysm size was $5.8 \mathrm{~mm}$ (range 1.1-16.9 mm). A total of 10 aneurysms showed enhancement on VWl; 5 ruptured aneurysms showed enhancement, and 1 unruptured but symptomatic aneurysm showed enhancement on VWI and ruptured 1 day later. Four unruptured aneurysms showed enhancement. In $6(60 \%)$ of the 10 enhanced aneurysms, intraluminal diameters appeared notably smaller ( $\geq 0.8 \mathrm{~mm}$ smaller) on contrast-enhanced VWI compared to their appearance on multiple overlapping thin slab acquisition time of flight (MOTSA-TOF) MRA and/or precontrast VWI, suggesting that enhancement was at least partially in the aneurysm lumen itself.

CONCLUSIONS Several factors other than the hypothesized inflammatory response contribute to aneurysm wall enhancement. In 60\% of the cases in this study, enhancement was at least partially caused by slow intraaneurysmal flow, leading to pseudo-enhancement of the aneurysm wall. Notwithstanding, there seems to be clinical value in differentiating ruptured from unruptured aneurysms using VWI, but the hypothesis that we image the inflammatory cell infiltration in the aneurysm wall is not yet confirmed.

https://thejns.org/doi/abs/10.3171/2019.4.FOCUS19236

KEYWORDS intracranial aneurysms; vessel wall imaging; enhancement

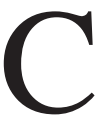
ONVENTIONAL imaging techniques such as CT, fluidattenuated inversion recovery (FLAIR) MRI, and susceptibility-weighted imaging (SWI) are being used in the acute setting of hemorrhagic stroke to detect subarachnoid hemorrhage (SAH). ${ }^{16,23}$ In cases of multiple intracranial aneurysms, the spatial relationship of the aneurysm with hemorrhage is the most solid indicator of the ruptured aneurysm (e.g., a lobar hematoma close to the aneurysm). However, for patients with a diffuse bleeding pattern or even the absence of blood on imaging (cerebro-

ABBREVIATIONS ACoA = anterior communicating artery; CSF = cerebrospinal fluid; FLAIR = fluid-attenuated inversion recovery; ICA = internal carotid artery; MCA = middle cerebral artery; MOTSA-TOF = multiple overlapping thin slab acquisition time of flight; $\mathrm{PCOA}=$ posterior communicating artery; SAH = subarachnoid hemorrhage; SWI = susceptibility-weighted imaging; $\mathrm{VWI}=$ vessel wall imaging.

SUBMITTED March 5, 2019. ACCEPTED April 12, 2019.

INCLUDE WHEN CITING DOI: 10.3171/2019.4.FOCUS19236. 
spinal fluid [CSF]-positive $\mathrm{SAH}$ ), it is often impossible to determine which aneurysm ruptured. And while size and morphology play a role in therapeutic decision-making, in cases in which rupture assessment is still inconclusive, the surgeon may end up treating all aneurysms, resulting in an increased treatment risk.

Histological analysis has shown significant differences in the composition of the aneurysm wall with a related difference in rupture rate. ${ }^{4}$ The exact mechanisms behind aneurysm rupture are not completely understood, and whether inflammation triggers aneurysm rupture or is the consequence of rupture is unknown. ${ }^{22}$ An increase in macrophage, T-cell, and leukocyte infiltration in the aneurysm wall has been associated with rupture, which has been the rationale for studying the aneurysm wall with vessel wall imaging (VWI). Recently, VWI is increasingly performed for its potential value in identifying ruptured aneurysms. ${ }^{7,15,18}$ It has been suggested that enhancement after contrast agent administration relates to inflammation in the aneurysm wall of unstable or ruptured aneurysms; therefore, VWI may assist in identifying ruptured aneurysms. ${ }^{8,20}$ However, aneurysm wall enhancement can be induced by several mechanisms other than this inflammatory response, such as the development of vasa vasorum, atherosclerosis, and intramural hematoma. ${ }^{2,8,9,19}$ In addition, intraaneurysmal flow has a completely different pattern than the laminar flow in the intracranial vessels, and this has been acknowledged to be an important pitfall in correctly appraising enhancement of the aneurysm wall in clinical practice. ${ }^{6,14}$

In this clinical report, we consider possible underlying mechanisms contributing to aneurysm wall enhancement by evaluating imaging data obtained using multiple modalities in patients suspected of having an SAH.

\section{Methods}

Approval from the local medical ethics committee was obtained for data collection and analysis of a prospective registry of SAH patients. Furthermore, as part of the General Data Protection Regulation (European Union), patients were asked permission to use clinical and imaging data for research purposes.

Patients with symptoms of SAH who subsequently underwent VWI in the period between January 2017 and September 2018 were eligible for inclusion in this study. For each patient, extensive MRI studies were acquired including multiple overlapping thin slab acquisition time of flight (MOTSA-TOF), FLAIR, and SWI sequences. For VWI, we acquired 3D turbo spin-echo black-blood MRI scans with motion-sensitized driven-equilibrium preparation pulses (in-plane resolution $=0.6 \mathrm{~mm}$, slice thickness $=$ $0.8 \mathrm{~mm}, \mathrm{TE}=35 \mathrm{msec}, \mathrm{TR}=700 \mathrm{msec}$, flip angle $=80^{\circ}$ ). This MRI protocol was performed in patients in whom CT scanning had been inconclusive and additional information was essential for detecting the ruptured aneurysm.

The aneurysm location, size, hemorrhagic distribution, and presence of blood on FLAIR and/or SWI in the vicinity of the aneurysm were assessed to determine which aneurysm was the most likely one that ruptured. Furthermore, aneurysm wall enhancement was assessed to deter- mine its relationship with aneurysm rupture. We defined aneurysm wall enhancement as focal or circumferential hyperintensity along the aneurysm wall on postcontrast VWI. Enhancement along the aneurysm wall is not limited to enhancement of the wall itself but can also originate from regions within or outside the aneurysm wall.

Complementary imaging data (CT, DSA, MRI) were used to assess potential underlying enhancement mechanisms, such as aneurysm rupture, growth, atherosclerotic calcifications, adjacent cerebral veins, and slow intraaneurysmal flow. MOTSA-TOF MRA was used to assess the intraluminal aneurysm size, and VWI before and after contrast agent administration allowed measurement of the nonopacified part of the aneurysm lumen (RadiAnt DICOM Viewer 4.2.1). Intraluminal diameters were considered to be decreased when the diameter on contrastenhanced VWI appeared $\geq 0.8 \mathrm{~mm}$ (slice thickness of VWI) smaller than its appearance on precontrast VWI or MOTSA-TOF.

\section{Results}

Six patients with 14 aneurysms were included in this case series. The mean aneurysm size was $5.8 \mathrm{~mm}$ (range $1.1-16.9 \mathrm{~mm}$ ). Information regarding the presence of blood on CT or in the CSF, the presence of spatially correlated blood on FLAIR and/or SWI, and aneurysm enhancement on contrast-enhanced VWI is presented in Table 1. A total of 10 aneurysms showed enhancement on VWI; all ruptured aneurysms enhanced (5 lesions including the aneurysm in case 5, which ruptured 1 day after VWI), and 4 unruptured aneurysms showed enhancement. In 2 patients, each harboring 2 aneurysms, all 4 aneurysms showed enhancement (cases 1 and 4). In another patient with a total of 6 aneurysms (case 2), 3 aneurysms showed enhancement.

In 6(60\%) of the 10 enhancing aneurysms, intraluminal diameters appeared notably smaller $(\geq 0.8 \mathrm{~mm}$ smaller) on contrast-enhanced VWI compared to their appearance on MOTSA-TOF and/or precontrast VWI, suggesting that enhancement was at least partially in the aneurysm lumen itself (Table 2).

One blister aneurysm (case 3) of the internal carotid artery (ICA) showed enhancement probably related to an intramural hemorrhage. Furthermore, circumferential enhancement was observed in a coiled posterior communicating artery (PCoA) aneurysm (case 6), which was most likely caused by a hyperintense coil-related rim artifact.

All aneurysms depicted as the most likely cause of SAH were treated. In patients in whom additional aneurysms had been left untreated, no rupture occurred during a follow-up period of at least 12 months.

\section{Illustrative Cases Case 1}

A 60-year-old man presented with clinical signs of SAH. CT imaging showed interhemispheric subarachnoid blood, continuing in the sylvian fissure until the middle cerebral artery (MCA) bifurcation, resulting from a ruptured bilobar anterior communicating artery (ACoA) 
TABLE 1. Patient characteristics and imaging findings for six patients suspected of having an SAH

\begin{tabular}{|c|c|c|c|c|c|c|c|c|}
\hline $\begin{array}{l}\text { Case } \\
\text { No. }\end{array}$ & $\mathrm{SAH}^{*}$ & $\begin{array}{l}\text { No. } \\
\text { of } \\
\text { IAs }\end{array}$ & Location & $\begin{array}{l}\text { Aneurysm } \\
\text { Size }(\mathrm{mm})\end{array}$ & FLAIR & SWI & VWE & Proposed Enhancement Mechanism \\
\hline \multirow[t]{2}{*}{1} & \multirow[t]{2}{*}{$+(\mathrm{CT})$} & \multirow[t]{2}{*}{2} & $\mathrm{ACoA} \dagger$ & 7.4 & + & + & + & Aneurysm rupture; suboptimal slow flow suppression \\
\hline & & & MCA & 2.9 & + & + & $+1-$ & Unclear; already high signal on precontrast VWI \\
\hline \multirow[t]{6}{*}{2} & \multirow[t]{6}{*}{$+(\mathrm{CT})$} & \multirow[t]{6}{*}{6} & Basilar tip† & 16.9 & + & + & + & $\begin{array}{l}\text { Previously coiled; progressive recanalization; aneurysm rupture; subop- } \\
\text { timal slow flow suppression }\end{array}$ \\
\hline & & & Pericallosal artery & 4.6 & - & - & + & $\begin{array}{l}\text { Suboptimal slow flow suppression; aneurysm growth during FU prior to } \\
\text { VWI }\end{array}$ \\
\hline & & & AChA & 4.2 & + & + & - & \\
\hline & & & Distal ICA & 2.8 & + & + & + & $\begin{array}{l}\text { Previously coiled; stable recanalization; suboptimal slow flow suppres- } \\
\text { sion }\end{array}$ \\
\hline & & & OphA & 4.7 & - & - & - & \\
\hline & & & MCA & 1.1 & + & + & - & \\
\hline 3 & $+(\mathrm{CSF})$ & 1 & ICA† & 2.7 & - & - & + & Intramural hematoma (blister aneurysm) \\
\hline \multirow[t]{2}{*}{4} & \multirow[t]{2}{*}{$+(\mathrm{CT})$} & \multirow[t]{2}{*}{2} & $\mathrm{ACoA} \dagger$ & 3.7 & + & + & + & Aneurysm rupture; suboptimal slow flow suppression \\
\hline & & & OphA & 3.8 & + & - & + & $\begin{array}{l}\text { Hyperintense signal along entire ICA segment, including ophthalmic } \\
\text { aneurysm; suboptimal flow suppression }\end{array}$ \\
\hline 5 & - & 1 & ICA bifurcation & 14.8 & - & - & + & $\begin{array}{l}\text { Aneurysm ruptured } 1 \text { day after VWl; calcified/atherosclerotic aneurysm } \\
\text { wall }\end{array}$ \\
\hline \multirow[t]{2}{*}{6} & \multirow[t]{2}{*}{$+(\mathrm{CSF})$} & \multirow[t]{2}{*}{2} & $\mathrm{PCoA} \dagger$ & 7.0 & - & - & + & Coil-induced hyperintense rim artifact \\
\hline & & & Pericallosal artery & 3.9 & - & - & - & \\
\hline
\end{tabular}

+ = positive; - = negative; +/- = equivocal; $\mathrm{AChA}$ = anterior choroidal artery; FU = follow-up; OphA = ophthalmic artery; VWE = vessel wall enhancement.

For each aneurysm, information on the presence of blood on CT or in the CSF, the presence of spatially correlated blood on FLAIR and/or SWI, and enhancement of the aneurysm after contrast agent administration on VWI is presented.

* Text in parentheses indicates whether SAH was indicated by blood on CT or in the CSF.

† Ruptured.

aneurysm $(7.4 \mathrm{~mm})$. A second left-sided, 2.9-mm, broadbased MCA bifurcation aneurysm was present. Given the small MCA aneurysm size and the presence of interhemispheric blood, the ACoA aneurysm was considered to be the most likely site of rupture. Focal areas of enhancement were seen surrounding the ACoA aneurysm on contrastenhanced VWI (Fig. 1). Because a smaller diameter was measured on postcontrast VWI compared to its size on MOTSA-TOF imaging (Table 2), insufficient flow suppression could have contributed to this enhancement.

A hyperintense signal surrounding the small MCA aneurysm was observed on both pre- and postcontrast VWI. On FLAIR images, this small MCA aneurysm was surrounded by subarachnoid blood. The cause of the high signal intensity along the wall of the aneurysm is unclear: focal subarachnoid blood, venous flow, or wall enhancement. The aneurysm remained stable during 12 months of follow-up.

\section{Case 2}

A 57-year-old woman with a large, previously ruptured and coiled basilar tip aneurysm again presented with SAH. Interpretation of CT imaging was hampered because of severe coil artifacts, and CTA could not be used to determine the cause of bleeding. MRI was performed 1 day later, with FLAIR imaging and SWI revealing bilateral insular blood. An evident increase in size and recanalization of the basilar tip aneurysm was seen. VWI demonstrated enhancement both within the lumen and in the wall of the basilar aneurysm (Fig. 2B-C). Since the lumen diameter appeared notably smaller on contrast-enhanced VWI compared to its size on precontrast VWI and MOTSATOF (Table 2), insufficient flow suppression could have resulted in an overestimation of wall enhancement.

An additional pericallosal artery aneurysm with slight growth over 4 years also showed wall enhancement (Fig. 2D-E), with a definite absence of subarachnoid blood on FLAIR imaging and SWI. DSA showed slow flow in the pericallosal artery aneurysm with stasis of blood in the aneurysm dome during the venous phase (Fig. 2F-G). Concomitant support for flow-related enhancement of the aneurysm lumen is the decrease in luminal diameter on contrast-enhanced VWI. The pericallosal aneurysm was treated after 12 months of follow-up.

A coiled distal ICA aneurysm with a small residual lumen after coiling also showed enhancement both in between the coils and in the small remnant. No enhancement was observed for the additional ophthalmic artery, MCA, and anterior choroidal artery aneurysms. It was decided to coil the basilar aneurysm because of the significant increase in size.

\section{Case 5}

A 42-year-old woman presented with an acute headache suspicious for SAH. Six weeks earlier, she had been diagnosed with an asymptomatic, large, right-sided ICA 
TABLE 2. Aneurysm diameter measurements on MOTSA-TOF, precontrast VWI, and postcontrast VWI

\begin{tabular}{|c|c|c|c|c|c|}
\hline \multirow{2}{*}{$\begin{array}{l}\text { Case } \\
\text { No. }\end{array}$} & \multirow{2}{*}{$\begin{array}{l}\text { Aneurysm Location } \\
\text { (side) }\end{array}$} & \multirow{2}{*}{$\begin{array}{l}\text { Max Aneurysm Diameter } \\
\text { on MOTSA-TOF }(\mathrm{mm})\end{array}$} & \multicolumn{3}{|c|}{ Diameter Measurements (mm), Matched Slices } \\
\hline & & & MOTSA-TOF & Precontrast VWI & Postcontrast VWI \\
\hline \multirow[t]{2}{*}{1} & $\mathrm{ACoA}$ & 7.4 & 6.4 & 6.1 & $5.6^{*}$ \\
\hline & $\operatorname{MCA}(\mathrm{It})$ & 2.9 & 2.9 & 2.7 & $2.5 \dagger$ \\
\hline \multirow[t]{6}{*}{2} & Basilar tipł & 16.9 & 10.9 & 9.7 & $8.6^{*}$ \\
\hline & Pericallosal artery & NA & NA & 4.6 & $3.8^{*}$ \\
\hline & $\mathrm{AChA}(\mathrm{rt})$ & 4.2 & 2.9 & 2.9 & 2.6 \\
\hline & Distal ICA (rt) $\ddagger$ & 2.8 & 2.8 & 2.8 & $0.8^{*}$ \\
\hline & OphA (It) & 4.7 & 4.2 & 3.8 & 3.7 \\
\hline & $\operatorname{MCA}(\mathrm{It})$ & 1.1 & 1.1 & 0.9 & 0.7 \\
\hline 3 & ICA (rt) & 2.7 & 2.1 & NA & $1.6 \dagger$ \\
\hline \multirow[t]{2}{*}{4} & $\mathrm{ACoA}$ & 3.7 & 2.9 & 2.9 & $1.0^{*}$ \\
\hline & OphA (It) & 3.8 & 3.8 & 3.7 & $2.1^{*}$ \\
\hline 5 & ICA bifurcation (rt) & 14.8 & 14.8 & NA & $14.8 \dagger$ \\
\hline \multirow[t]{2}{*}{6} & $\mathrm{PCoA}(\mathrm{rt}) \S$ & 7.0 & 6.3 & NA & $6.3 \dagger$ \\
\hline & Pericallosal artery & 3.9 & 3.6 & NA & 3.6 \\
\hline $\begin{array}{l}\text { NA }=\text { nc } \\
{ }^{*} \text { Enhar } \\
\dagger \text { Enhar } \\
\text { † Meas } \\
\text { S Meas }\end{array}$ & $\begin{array}{l}\text { plicable. } \\
\text { ent with decreased lu } \\
\text { hent on postcontrast } \\
\text { eent of residual aneur } \\
\text { eent of coiled aneurys }\end{array}$ & $\begin{array}{l}\text { nal diameter ( } \geq 0.8 \mathrm{~mm} \text { small } \\
\text { m. } \\
\text { n lumen after coiling. }\end{array}$ & & & \\
\hline
\end{tabular}

bifurcation aneurysm $(14.8 \mathrm{~mm})$ with coarse atherosclerotic calcifications at the region of the aneurysm neck and small spots of calcifications along the aneurysm wall. CT imaging and lumbar puncture at the acute admission did not reveal an SAH. In accordance, FLAIR and SW images did not show any signs of bleeding. On VWI, circumferential aneurysm wall enhancement was observed (Fig. 3). One day later the patient had a severe SAH, and despite emergency treatment she died from complications. Enhancement of the aneurysm prior to rupture might have indicated instability of the aneurysm; however, atherosclerosis and inflammatory reactions in the thickened aneurysm wall might have also contributed to aneurysm wall enhancement.

\section{Case 6}

A 58-year-old woman presented with an acute headache. CT imaging did not demonstrate subarachnoid blood; however, lumbar puncture was positive for SAH. CTA revealed a right-sided, 7.0-mm PCoA aneurysm, which was subsequently coiled. An additional small pericallosal artery aneurysm $(3.9 \mathrm{~mm})$ was identified during angiography. Given the CT scan, rupture of this additional aneurysm could not be excluded. Additional MRI revealed neither blood or hemosiderin in the vicinity of this aneurysm, nor VWI enhancement of the pericallosal aneurysm. Therefore, the PCoA was considered to be the most likely site of rupture. This PCoA aneurysm showed enhancement of the aneurysm wall; however, increased signal intensity was already present on the precontrast T1-weighted MR images (Fig. 4). A coiling-induced hyperintense rim artifact most likely contributed to this high signal intensity. ${ }^{13}$ The pericallosal aneurysm was left untreated, and followup revealed a stable aspect of the aneurysm at 12 months.

\section{Discussion}

In all SAH patients in this case series, wall enhancement of the ruptured aneurysm was observed. Four of 8 unruptured aneurysms also showed wall enhancement. In $60 \%$ of the enhanced aneurysms, the aneurysm lumen appeared notably smaller on contrast-enhanced VWI compared to its size on MOTSA-TOF MRA and noncontrast-enhanced VWI. This strongly suggests that slow flow at the inner border of the aneurysm wall contributes to - and thus may lead to an overestimation of-aneurysm wall enhancement. Additionally, atherosclerosis $(n=1)$, coiling-induced hyperintense rim artifact $(n=1)$, and intramural hematoma $(n=1)$ contributed to aneurysm wall enhancement. Our data suggest that VWI may be highly sensitive but has limited specificity for detecting ruptured aneurysms. Therefore, aneurysm assessment should not rely solely on VWI.

Several studies have related aneurysm wall enhancement to rupture status. VWI after SAH in a case series of five patients demonstrated enhancement of the aneurysm wall in all ruptured aneurysms, while none of the unruptured aneurysms enhanced..$^{15}$ Concordantly, vessel wall assessment in three large cohorts with a total of 108 ruptured aneurysms and 302 unruptured aneurysms showed a correlation between enhancement of the aneurysm wall and rupture status. ${ }^{17,18,24}$ One of these studies reported that partial wall enhancement was solely observed at irregular regions and daughter sacs of aneurysms. ${ }^{24}$ Because aneurysm irregularity has been related to rupture status, some have hypothesized that endothelial damage and inflammation are the likely causes of aneurysm enhancement. ${ }^{10}$ However, these irregular regions and daughter sacs are very susceptible to different, much slower flow patterns, 

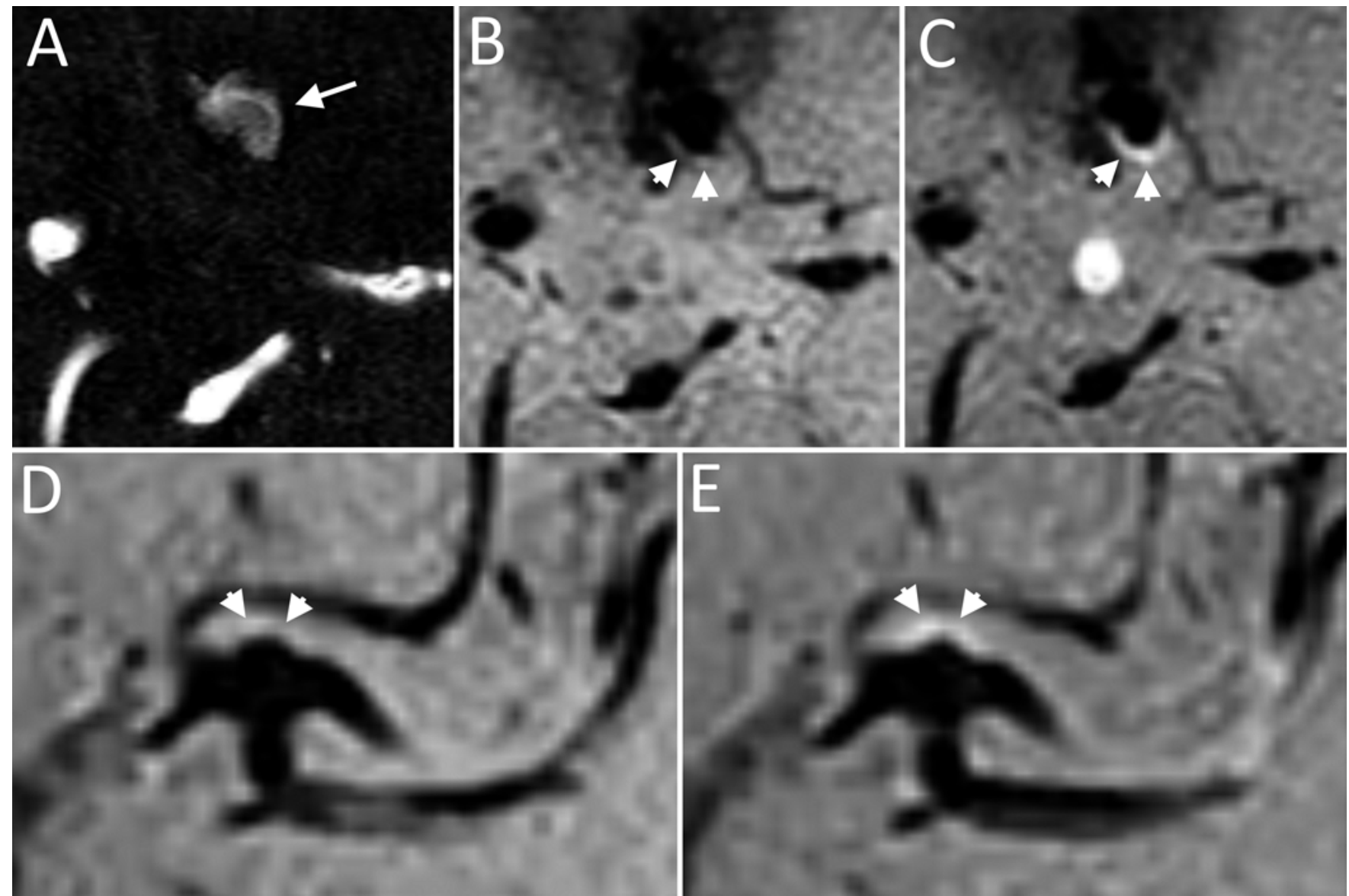

FIG. 1. Case 1. A: Axial MOTSA-TOF MR angiogram of a ruptured bilobar ACoA aneurysm (arrow). B: On a precontrast VW image, arrows indicate the ACoA aneurysm wall. C: Contrast-enhanced VW image with local aneurysm wall enhancement (arrows). D: Hyperintense signal (arrows) surrounding the small MCA aneurysm on precontrast VWI. E: The hyperintense signal (arrows) became more evident on postcontrast VWI.

which may lead to insufficient flow suppression within the aneurysm. As we observed in some of our cases by measuring the internal luminal diameter of the aneurysm on MOTSA-TOF and pre- and postcontrast VWI, the luminal diameter appears smaller on contrast-enhanced VWI. This is most likely caused by slow adherent flow close to the inner border of the aneurysm wall, which can contribute to pseudo-enhancement of the aneurysm wall. Looking at case examples from other publications on VWI in aneurysms suggests that this flow-related enhancement inside the aneurysm lumen is not a rare phenomenon. $3,15,17$

Besides its relation to rupture status, enhancement has also been observed more frequently in unstable than in stable intracranial aneurysms. ${ }^{3}$ Edjlali et al. defined unstable aneurysms as recently (within 24 hours) ruptured, symptomatic, or evolving with changing morphology over 6 months. In another publication focusing on unruptured aneurysms only, circumferential aneurysm wall enhancement was observed more frequently in symptomatic (70\%) than in asymptomatic (27\%) aneurysms. ${ }^{5}$ Patients with symptomatic aneurysms in that study had presented with either sentinel headaches or third nerve palsy. Occasionally, wall enhancement on VWI has been associated with inflammatory cell invasion in unruptured aneurysms, as shown by Larsen and colleagues using histological analysis. ${ }^{8}$ Moreover, those authors did not observe enhancement in aneurysms without histological signs of inflammation.

The suggestion that VWI can be used as an indirect marker of vessel wall inflammation has been made by several authors, and the relation to inflammatory processes in the aneurysm wall as found with histopathology is easily made. ${ }^{3,15,17}$ Frösen et al. categorized intracranial aneurysms into four different types according to wall histology, with associated differences in the rupture rate. ${ }^{4}$ Endothelialized wall with linearly organized smooth-muscle cells showed the lowest rupture rate (42\% ruptured), whereas aneurysms with an extremely thin thrombosis-lined hypocellular wall were all ruptured. A study of postmortem and intraoperatively acquired intracranial aneurysms has shown that aneurysm walls can be very thin, measuring between 0.02 and $0.50 \mathrm{~mm} .{ }^{21}$ MRI of the aneurysm wall can be challenging because of image resolution; the best obtainable image resolution using 3-T MRI is usually around $0.8 \mathrm{~mm}$, which is insufficient to visualize the average wall thickness of an aneurysm. However, a recent study correlated histopathology and 7-T MRI of aneurysms, showing that 7-T MRI can demonstrate intracranial aneurysm walls and assess variation in wall thickness.? 

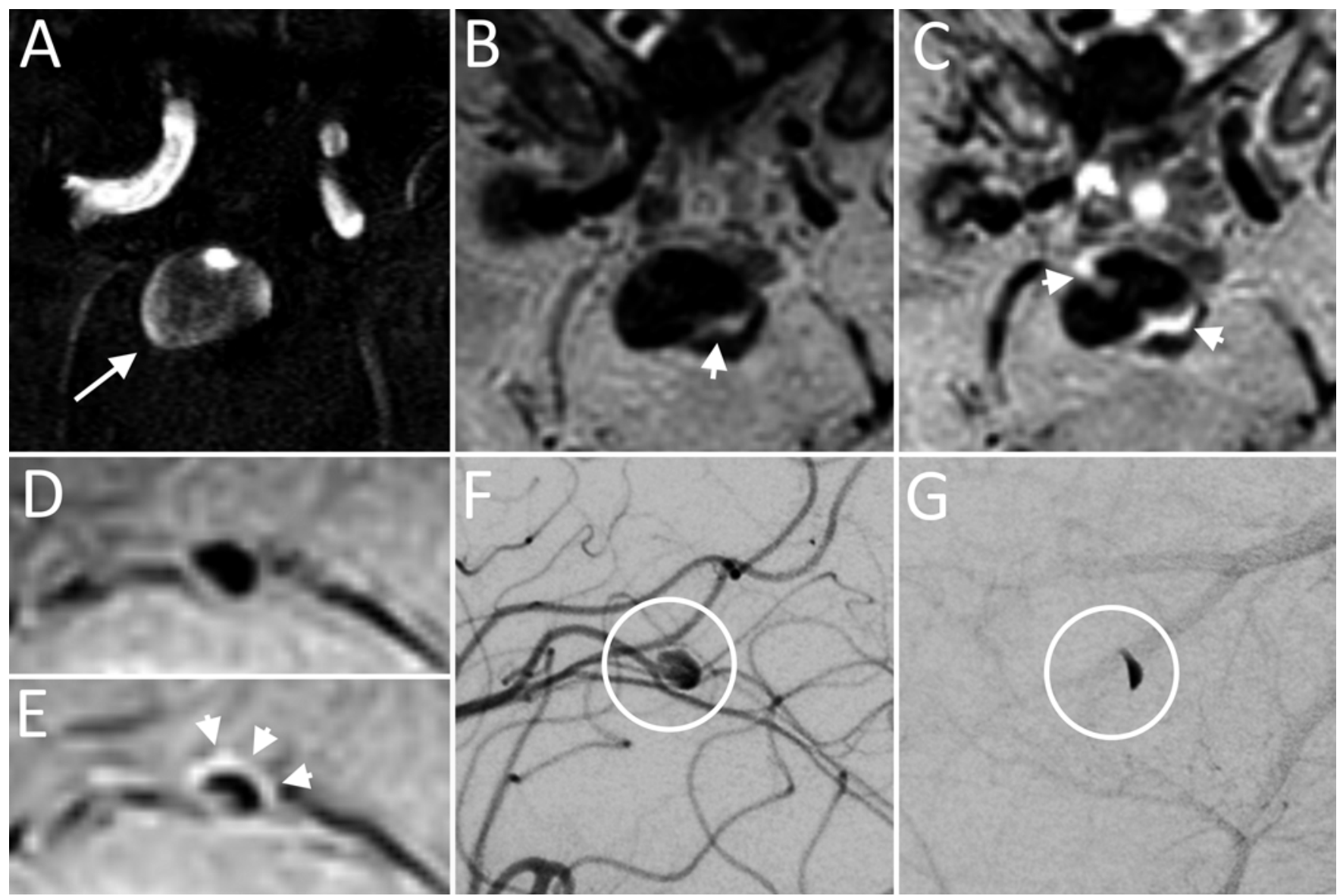

FIG. 2. Case 2. A: Axial MOTSA-TOF MR angiogram of a ruptured, recurrent basilar tip aneurysm (arrow). B: Hyperintense MR signal (arrow) surrounding the coil mass on precontrast VWI. C: The signal surrounding the coil mass enhanced on postcontrast VWI. Furthermore, intraluminal enhancement (arrows) was observed. D and E: Sagittal pre- and postcontrast VWI showed a growing pericallosal artery aneurysm with clear circumferential enhancement (arrows). F: DSA revealed the pericallosal aneurysm.

G: Intraaneurysmal stasis of contrast agent in the venous phase, indicating slow intraaneurysmal flow.

Added to the limitation in resolution are the pulsation artifacts on MRI, which degrade image quality and incorrectly increase aneurysm wall thickness. These pulsation artifacts can be overcome by using cardiac gating during VWI. Another limitation of VWI is that an intraluminal thrombus may hinder reliable assessment of aneurysm wall thickness as the clot itself can already show high signal intensity on precontrast VWI. When using VWI to show wall enhancement, a precontrast VWI sequence is mandatory to exclude false-positive cases. Another pitfall in VWI can be enhancement of the venous structures close to the aneurysm.

An association between wall enhancement and aneurysm size in unruptured intracranial aneurysms has been shown in several studies.,11,12,26 However, a large aneurysm size hinders reliable assessment of aneurysm wall enhancement as lower velocities are to be expected in larger aneurysms. Wall-mimicking artifacts can be caused by insufficient flow suppression, which may lead to an overestimation of aneurysm wall enhancement with possible false-positive findings (pseudo-enhancement) in large aneurysms.

Correct delineation of the aneurysm wall can be very difficult when the wall lies close to the brain parenchyma. It has even been suggested that in ruptured aneurysms enhancement of the aneurysm wall corresponds to the interface between the aneurysm wall and surrounding brain tissue ${ }^{18}$ It has also been suggested that the vasa vasorum plays a role in aneurysm wall enhancement; however, histopathological confirmation is often lacking. ${ }^{19}$ Most of the studies on the presence of vasa vasorum in aneurysms have looked at giant and chronic fusiform aneurysms, but in a recent histological analysis, the presence of the vasa vasorum was noted in 2 of 14 patients with non-giant aneurysms of 14 and $6 \mathrm{~mm} .{ }^{8}$

How should the value of VWI in differentiating ruptured from unruptured aneurysms be judged-as it does seem to point to the ruptured aneurysm? First, irregular aneurysms with blebs have a higher rupture risk than more regular aneurysms. Stagnation of flow in these blebs with resulting flow-related enhancement is to be expected. Larger aneurysms have a higher risk of rupture, and the relation between aneurysm size and slow flow has already been acknowledged. Aneurysm wall enhancement has also been related to areas of low wall shear stress in a series of patients with ruptured and unruptured aneurysms. ${ }^{25}$ How- 


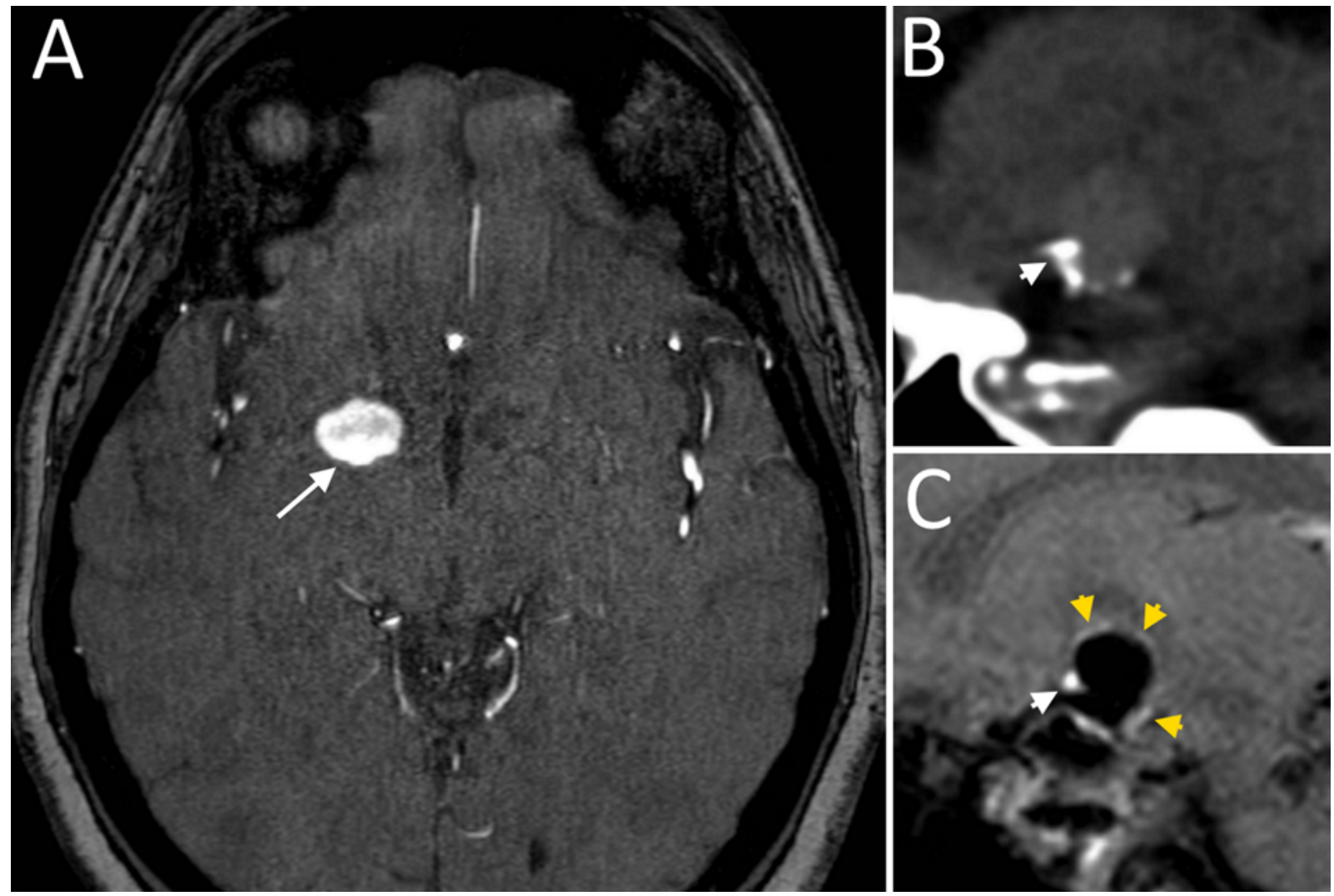

FIG. 3. Case 5. A: MOTSA-TOF MR angiogram of an unruptured ICA bifurcation aneurysm (arrow) 1 day prior to rupture. B: Sagittal $\mathrm{CT}$ image of the unruptured aneurysm with atherosclerotic calcifications (arrow) at the neck region of the aneurysm. C: Sagittal postcontrast VWI with a high degree of enhancement at the aneurysm neck region (white arrow) and circumferential wall enhancement (yellow arrows).
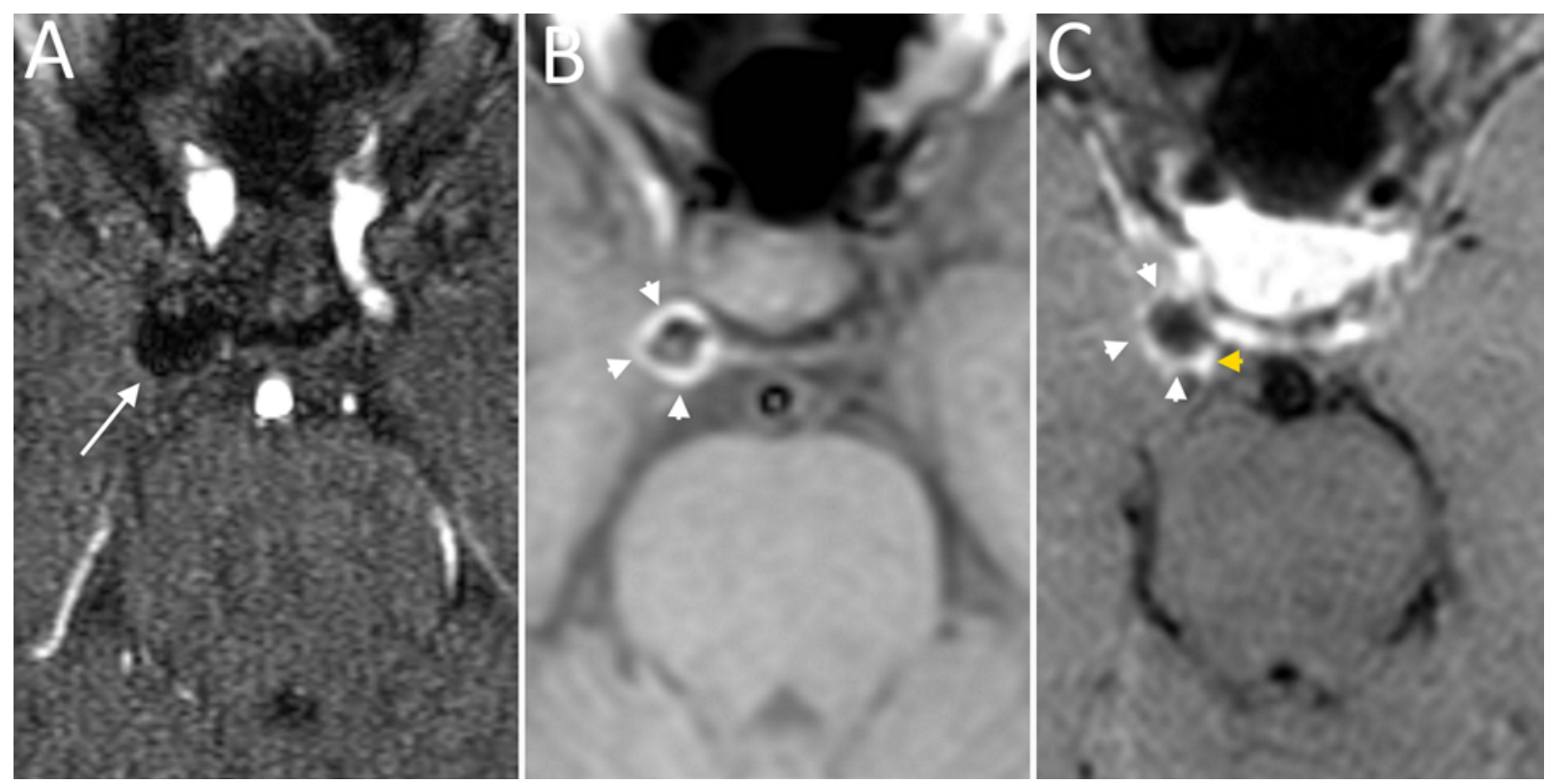

FIG. 4. Case 6. A: MOTSA-TOF MR angiogram obtained 4 days after coiling, showing a rim artifact of a ruptured PCoA aneurysm. B: Precontrast T1-weighted image showing a circumferential hyperintense signal (arrows). C: Postcontrast VWI with a circumferential hyperintense signal (white arrows). The cavernous sinus also shows enhancement (yellow arrow). 
ever, at least in one of the case examples in the study by Xiao et al., enhancement is seen not only in the wall of the aneurysm but also within the lumen of the aneurysm bleb. Important to note is that slow flow artifacts do not necessarily invalidate the relationship between enhancement and aneurysm stability since slow flow can be an indirect marker of wall inflammation. To confirm the relationship between inflammation and enhancement, histological assessment remains necessary.

\section{Conclusions}

Several factors other than the hypothesized inflammatory response contribute to aneurysm wall enhancement. In $60 \%$ of our cases, enhancement was at least partially caused by slow intraaneurysmal flow, leading to pseudoenhancement of the aneurysm wall. Notwithstanding, there seems to be clinical value in differentiating ruptured from unruptured aneurysms using VWI, but the hypothesis that we image the inflammatory cell infiltration in the aneurysm wall is not yet confirmed.

\section{Acknowledgments}

An unrestricted grant was provided to Amsterdam University Medical Centers, location AMC, by TWIN Foundation, who had no role in the study design; collection, analysis, or interpretation of the data; the writing of the report; or the decision to submit the paper for publication.

\section{References}

1. Backes D, Hendrikse J, van der Schaaf I, Algra A, Lindgren $\mathrm{AE}$, Verweij BH, et al: Determinants of gadolinium-enhancement of the aneurysm wall in unruptured intracranial aneurysms. Neurosurgery 83:719-725, 2018

2. Coutinho JM, Sacho RH, Schaafsma JD, Agid R, Krings T, Radovanovic I, et al: High-resolution vessel wall magnetic resonance imaging in angiogram-negative non-perimesencephalic subarachnoid hemorrhage. Clin Neuroradiol 27:175183,2017

3. Edjlali M, Guédon A, Ben Hassen W, Boulouis G, Benzakoun J, Rodriguez-Régent C, et al: Circumferential thick enhancement at vessel wall MRI has high specificity for intracranial aneurysm instability. Radiology 289:181-187, 2018

4. Frösen J, Piippo A, Paetau A, Kangasniemi M, Niemelä M, Hernesniemi J, et al: Remodeling of saccular cerebral artery aneurysm wall is associated with rupture: histological analysis of 24 unruptured and 42 ruptured cases. Stroke 35:22872293, 2004

5. Fu Q, Guan S, Liu C, Wang K, Cheng J: Clinical significance of circumferential aneurysmal wall enhancement in symptomatic patients with unruptured intracranial aneurysms: a high-resolution MRI study. Clin Neuroradiol 28:509-514, 2018

6. Kalsoum E, Chabernaud Negrier A, Tuilier T, Benaïssa A, Blanc R, Gallas S, et al: Blood flow mimicking aneurysmal wall enhancement: a diagnostic pitfall of vessel wall MRI using the postcontrast 3D turbo spin-echo MR imaging sequence. AJNR Am J Neuroradiol 39:1065-1067, 2018

7. Kleinloog R, Korkmaz E, Zwanenburg JJM, Kuijf HJ, Visser F, Blankena R, et al: Visualization of the aneurysm wall: a 7.0-tesla magnetic resonance imaging study. Neurosurgery 75:614-622, 2014

8. Larsen N, von der Brelie C, Trick D, Riedel CH, Lindner T, Madjidyar J, et al: Vessel wall enhancement in unruptured intracranial aneurysms: an indicator for higher risk of rup- ture? High-resolution MR imaging and correlated histologic findings. AJNR Am J Neuroradiol 39:1617-1621, 2018

9. Lindenholz A, van der Kolk AG, Zwanenburg JJM, Hendrikse J: The use and pitfalls of intracranial vessel wall imaging: how we do it. Radiology 286:12-28, 2018

10. Lindgren AE, Koivisto T, Björkman J, von Und Zu Fraunberg M, Helin K, Jääskeläinen JE, et al: Irregular shape of intracranial aneurysm indicates rupture risk irrespective of size in a population-based cohort. Stroke 47:1219-1226, 2016

11. Liu P, Qi H, Liu A, Lv X, Jiang Y, Zhao X, et al: Relationship between aneurysm wall enhancement and conventional risk factors in patients with unruptured intracranial aneurysms: a black-blood MRI study. Interv Neuroradiol 22:501-505, 2016

12. Lv N, Karmonik C, Chen S, Wang X, Fang Y, Huang Q, et al: Relationship between aneurysm wall enhancement in vessel wall magnetic resonance imaging and rupture risk of unruptured intracranial aneurysms. Neurosurgery 84:E385-E391, 2019

13. Majoie CBLM, Sprengers ME, van Rooij WJJ, Lavini C, Sluzewski M, van Rijn JC, et al: MR angiography at 3T versus digital subtraction angiography in the follow-up of intracranial aneurysms treated with detachable coils. AJNR Am J Neuroradiol 26:1349-1356, 2005

14. Mandell DM, Mossa-Basha M, Qiao Y, Hess CP, Hui F, Matouk $\mathrm{C}$, et al: Intracranial vessel wall MRI: principles and expert consensus recommendations of the American Society of Neuroradiology. AJNR Am J Neuroradiol 38:218-229, 2017

15. Matouk CC, Mandell DM, Günel M, Bulsara KR, Malhotra A, Hebert R, et al: Vessel wall magnetic resonance imaging identifies the site of rupture in patients with multiple intracranial aneurysms: proof of principle. Neurosurgery 72:492-496, 2013

16. Mitchell P, Wilkinson ID, Hoggard N, Paley MNJ, Jellinek DA, Powell T, et al: Detection of subarachnoid haemorrhage with magnetic resonance imaging. J Neurol Neurosurg Psychiatry 70:205-211, 2001

17. Nagahata S, Nagahata M, Obara M, Kondo R, Minagawa N, Sato S, et al: Wall enhancement of the intracranial aneurysms revealed by magnetic resonance vessel wall imaging using three-dimensional turbo spin-echo sequence with motionsensitized driven-equilibrium: a sign of ruptured aneurysm? Clin Neuroradiol 26:277-283, 2016

18. Omodaka S, Endo H, Niizuma K, Fujimura M, Inoue T, Sato $\mathrm{K}$, et al: Quantitative assessment of circumferential enhancement along the wall of cerebral aneurysms using MR imaging. AJNR Am J Neuroradiol 37:1262-1266, 2016

19. Portanova A, Hakakian N, Mikulis DJ, Virmani R, Abdalla WMA, Wasserman BA: Intracranial vasa vasorum: insights and implications for imaging. Radiology 267:667-679, 2013

20. Shimonaga K, Matsushige T, Ishii D, Sakamoto S, Hosogai M, Kawasumi T, et al: Clinicopathological insights from vessel wall imaging of unruptured intracranial aneurysms. Stroke 49:2516-2519, 2018

21. Suzuki J, Ohara H: Clinicopathological study of cerebral aneurysms. Origin, rupture, repair, and growth. J Neurosurg 48:505-514, 1978

22. Tulamo R, Frösen J, Hernesniemi J, Niemelä M: Inflammatory changes in the aneurysm wall: a review. J Neurointerv Surg 2:120-130, 2010

23. Verma RK, Kottke R, Andereggen L, Weisstanner C, Zubler C, Gralla J, et al: Detecting subarachnoid hemorrhage: comparison of combined FLAIR/SWI versus CT. Eur J Radiol 82:1539-1545, 2013

24. Wang G, Wen L, Lei S, Ran Q, Yin J, Gong Z, et al: Wall enhancement ratio and partial wall enhancement on MRI associated with the rupture of intracranial aneurysms. J Neurointerv Surg 10:566-570, 2018 
25. Xiao W, Qi T, He S, Li Z, Ou S, Zhang G, et al: Low wall shear stress is associated with local aneurysm wall enhancement on high-resolution MR vessel wall imaging. AJNR Am J Neuroradiol 39:2082-2087, 2018

26. Zhu C, Wang X, Degnan AJ, Shi Z, Tian B, Liu Q, et al: Wall enhancement of intracranial unruptured aneurysm is associated with increased rupture risk and traditional risk factors. Eur Radiol 28:5019-5026, 2018

\section{Disclosures}

The authors report no conflict of interest concerning the materials or methods used in this study or the findings specified in this paper.

\section{Author Contributions}

Conception and design: van den Berg, Cornelissen, Leemans. Acquisition of data: van den Berg, Cornelissen. Analysis and interpretation of data: all authors. Drafting the article: van den Berg, Cornelissen. Critically revising the article: all authors.

\section{Correspondence}

René van den Berg: Amsterdam UMC, Amsterdam, The Netherlands.r.vdberg@amc.uva.nl. 